\title{
The chemical composition of the peculiar companion to the millisecond pulsar in NGC $6397^{\star}$
}

\author{
E. Sabbi ${ }^{1}$, R. G. Gratton ${ }^{2}$, A. Bragaglia ${ }^{3}$, F. R. Ferraro ${ }^{1}$, A. Possenti ${ }^{4}$, F. Camilo ${ }^{5}$, and N. D’ Amico ${ }^{4,6}$ \\ 1 Dipartimento di Astronomia Università di Bologna, via Ranzani 1, 40127 Bologna, Italy \\ 2 INAF-Osservatorio Astronomico di Padova, vicolo dell'Osservatorio 5, 35122 Padova, Italy \\ 3 INAF-Osservatorio Astronomico di Bologna, via Ranzani 1, 40126 Bologna, Italy \\ ${ }^{4}$ INAF-Osservatorio Astronomico di Cagliari, Loc. Poggio dei Pini, Strada 54, 09012 Capoterra, Italy \\ 5 Columbia Astrophysics Laboratory, Columbia University, 550 West 120th Street, New York, NY 10027, USA \\ ${ }^{6}$ Dipartimento di Fisica Università di Cagliari, Cittadella Universitaria, 09042 Monserrato, Italy
}

Received 19 June 2003 / Accepted 19 September 2003

\begin{abstract}
We present the chemical composition of the bright companion to the millisecond pulsar J1740-5340 in NGC 6397, based on high resolution spectra. Though the large rotation velocity of the star broadens the lines and complicates the analysis, the derived abundances are found fully compatible with those of normal unperturbed stars in NGC 6397, with the exception of a few elements ( $\mathrm{Li}, \mathrm{Ca}$, and $\mathrm{C}$ ). The lack of $\mathrm{C}$ suggests that the star has been peeled down to regions where incomplete CNO burning occurs, favouring a scenario where the companion is a turn-off star which has lost most of its mass. In addition we found an unexpected large $\mathrm{Li}$ abundance, which suggests that fresh ${ }^{7} \mathrm{Li}$ has been produced on the stellar surface.
\end{abstract}

Key words. Galaxy: globular clusters: individual: NGC 6397 - stars: evolution - stars: abundances - stars: pulsars: individual: PSR J1740-5340 - techniques: spectroscopic

\section{Introduction}

PSR J1740-5340 is a binary eclipsing millisecond pulsar (MSP) discovered by D'Amico et al. (2001a, 2001b) in the globular cluster NGC 6397. A bright and variable star, with anomalous red colours (hereafter COM J1740-5340), was identified by Ferraro et al. (2001) as the companion to the MSP. This is the first observed example of a MSP companion whose light curve is dominated by ellipsoidal variations, suggestive of a tidally distorted star, which almost completely fills (and is still overflowing) its Roche lobe.

Binary evolution calculations (e.g. Tauris \& Savonije 1999; Podsiadlowski et al. 2002) and the optical detections of a few sources in the Galactic field (e.g. Hansen \& Phinney 1998; Stappers et al. 2001) show that the most common companion to a binary MSP is either a white dwarf or a very light $\left(0.01-0.03 M_{\odot}\right)$ almost exhausted not fully degenerate star. In the crowded stellar environment of a globular cluster, other kinds of companion are also possible, resulting from dynamical encounters in the cluster core. For example, the MSP 47 Tuc-W in 47 Tucanae is orbited by a companion whose position in the colour-magnitude diagram (CMD) suggests that it is a main

Send offprint requests to: E. Sabbi,

e-mail: sabbi@bo.astro.it

* Based on observations collected at the European Southern Observatory, Chile, under proposal number 69.D-0264. sequence (MS) star heated by the pulsar radiation flux (Edmonds et al. 2002).

None of these hypotheses fit with the observed features of COM J1740-5340: it is too luminous to be a white dwarf ( $V \sim 16.6$, comparable to the turn-off stars of NGC 6397, Taylor et al. 2001; Ferraro et al. 2001); its mass $\left(\sim 0.3 M_{\odot}\right.$, Ferraro et al. 2003, hereafter Paper I; Kaluzny et al. 2003) is too high for a light stellar companion; finally, if originally it was a MS star, its anomalous red colour would imply a bloating of the atmospheric regions much larger than predicted by any available model (e.g. Podsiadlowski 1991; D'Antona et al. 1996). As a consequence, many intriguing scenarios were proposed in order to explain the nature of this binary system (see Possenti 2002; Orosz \& van Kerwijk 2002; Grindlay et al. 2002 for reviews).

The unusual brightness of COM J1740-5340 allows in principle a detailed study of its chemical composition and derivation of the structural parameters of this binary system, opening new possibilities in studying the origin of MSPs in clusters. In this framework we started a spectroscopic campaign with ESO telescopes; this paper is the third in a series which reports on these observations. In Paper I we presented the first results based on the high resolution spectra: we provided the radial velocity curve, the mass ratio, the determination of the component masses, a preliminary evaluation of the metallicity and a discussion of the heating of the companion 
surface due to the impinging MSP flux. In Sabbi et al. (2003, Paper II) we showed the complex structure of the $\mathrm{H} \alpha$ line (deriving important information on the mass loss) and discussed the unexpected presence of $\mathrm{He}$ I lines, implying the existence of a very narrow heated region on the companion surface. This paper focuses on the chemical abundance analysis. The observational data are presented in Sect. 2, while in Sect. 3 we report on the rotational velocity, the atmospheric parameters and the equivalent widths of the observed spectral lines. Section 4 is devoted to the determination of the actual element abundances, which are discussed in Sect. 5.

\section{Observations and data reduction}

Eight high resolution spectra of COM J1740-5340, taken at different phases and covering the whole orbital period of the binary system $(P \sim 1.35$ days $)$ were acquired with the Ultraviolet-Visual Echelle Spectrograph (UVES) mounted at the Kueyen 8 m-telescope (UT2) of the ESO Very Large Telescope on Cerro Paranal (Chile). More details on the observational strategy can be found in Paper I. COM J1740-5340 is located in a crowded region, hence seeing conditions are relevant to minimize contamination from nearby objects: all the spectra (but one) were taken with seeing between 0.9" and $1.2^{\prime \prime}$. Our spectra cover, with some gaps, the wavelength range 3280-6725 $\AA$, at a resolution $R \sim 40000$; the $S / N$ of the individual spectra varies, but it is typically about 30-35 near $\mathrm{H} \alpha$.

In the following analysis we started with the onedimensional, wavelength calibrated spectra produced by the UVES pipeline (Ballester et al. 2000). All our abundance analysis is applied to the sum of two spectra acquired near the orbital quadratures (at orbital phases $\sim 0.02$, and 0.56 respectively), when the radial velocities are highest and the larger Doppler shifts ensure that contamination of lines by the sky and/or by other cluster stars is negligible (Paper I). After excision of cosmic rays and shift to zero radial velocities, we combined the spectra and rebinned them with $0.3 \AA$ resolution in order to enhance the $S / N(\sim 60$ near $\mathrm{H} \alpha)$ up to the level required by the purposes of the present paper; there is no loss of information in doing so, given the width of the lines.

\section{Analysis and error estimates}

\subsection{Rotation velocity}

The lines of COM J1740-5340 are greatly broadened by the rotation velocity of the star. In order to evaluate this quantity, we exploited the spectra of three subgiants (SGB) in NGC 6397 (i.e. stars with similar temperature and gravity). They were observed using a UVES configuration similar to ours within a project devoted to study the chemical composition of NGC 6397 (Gratton et al. 2001, hereafter G01). The positions of the three aforementioned subgiants and COM J1740-5340 are highlighted in the CMD shown in Fig. 1.

In order to derive the rotation velocity we selected the subgiant observed with the highest $S / N$ ratio, namely \# 793 in G01

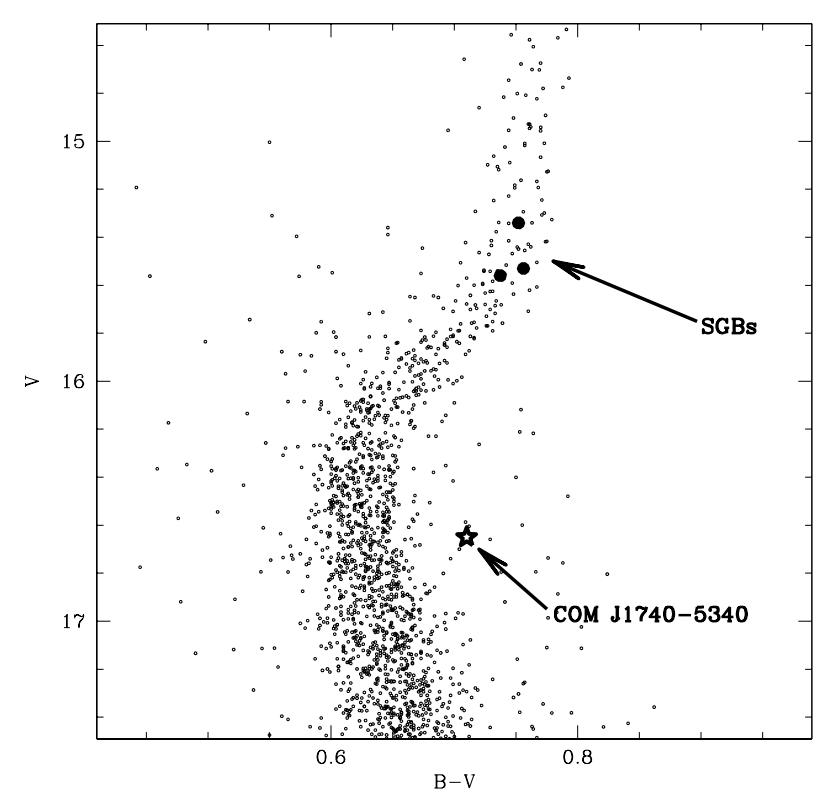

Fig. 1. Position in the colour-magnitude diagram of the three subgiant stars examined in G01 (filled dots) and of COM J1740-5340 (star). COM J1740-5340 $B$ and $V$ magnitudes are taken from Kaluzny et al. (2003, Table 1, in quadrature).

$\left([\mathrm{Fe} / \mathrm{H}]^{1}=-2.04, T_{\text {eff }}=5478 \mathrm{~K}, \log g=3.4, S / N \sim 100\right)$. It represents a very good template for a "not rotating" star: a firm upper limit to the projected rotation velocity has been recently set at $V \sin i \leq 3.7 \mathrm{~km} \mathrm{~s}^{-1}$ (Lucatello \& Gratton 2003).

A cross-correlation technique was applied to compare the spectrum of the selected subgiant with that of COM J1740-5340. This technique gives an upper limit to the real rotation velocity, because the cross correlation peak is widened by factors other than rotation (line width of the template star; micro- and macro-turbulence for the program star; instrumental profile). However, in our case these other widening factors are much smaller than those due to rotation of COM J1740-5340: the FWHM of the profiles of the template lines is $10.5 \mathrm{~km} \mathrm{~s}^{-1}$; typical macro-turbulent velocity for subgiants of NGC 6397 is $6.7 \mathrm{~km} \mathrm{~s}^{-1}$ (Lucatello \& Gratton 2003); the microturbulent velocity for COM J1740-5340 is about $1.0 \mathrm{~km} \mathrm{~s}^{-1}$ (see below); and finally, the instrumental broadening is about $6 \mathrm{~km} \mathrm{~s}^{-1}$. When we sum in quadrature all these terms, the total contribution to line width is $13.9 \mathrm{~km} \mathrm{~s}^{-1}$. We can then safely assume that the rotational velocity of COM J1740-5340 (expected to be about $50 \mathrm{~km} \mathrm{~s}^{-1}$ ) dominates the width of the cross-correlation peak.

As the blue part of the spectra is richer in lines and lacks telluric features, three different portions of this spectral region (namely $\lambda \lambda$ 4120-4180, 4120-4310, and 4361-4441 $⿱$ ) were selected, avoiding strong lines like $\mathrm{Ca}$ II $\mathrm{H}$ and $\mathrm{K}$, and Balmer. We have determined the FWHM (i.e. the rotational velocity) of the three cross-correlation peaks, fitting them with a Dirac $\delta$ function broadened by rotation effects, assuming a gray atmosphere, a normal limb-darkening law (coefficient equal to 0.6 , appropriate for stars of this temperature), and several

\footnotetext{
${ }^{1} \mathrm{We}$ adopt the usual spectroscopic notation that $[\mathrm{A} / \mathrm{B}]=$ $\log _{10}\left(N_{\mathrm{A}} / N_{\mathrm{B}}\right)_{\text {star }}-\log _{10}\left(N_{\mathrm{A}} / N_{\mathrm{B}}\right)_{\odot}$ for element $\mathrm{A}$ and $\mathrm{B}$.
} 


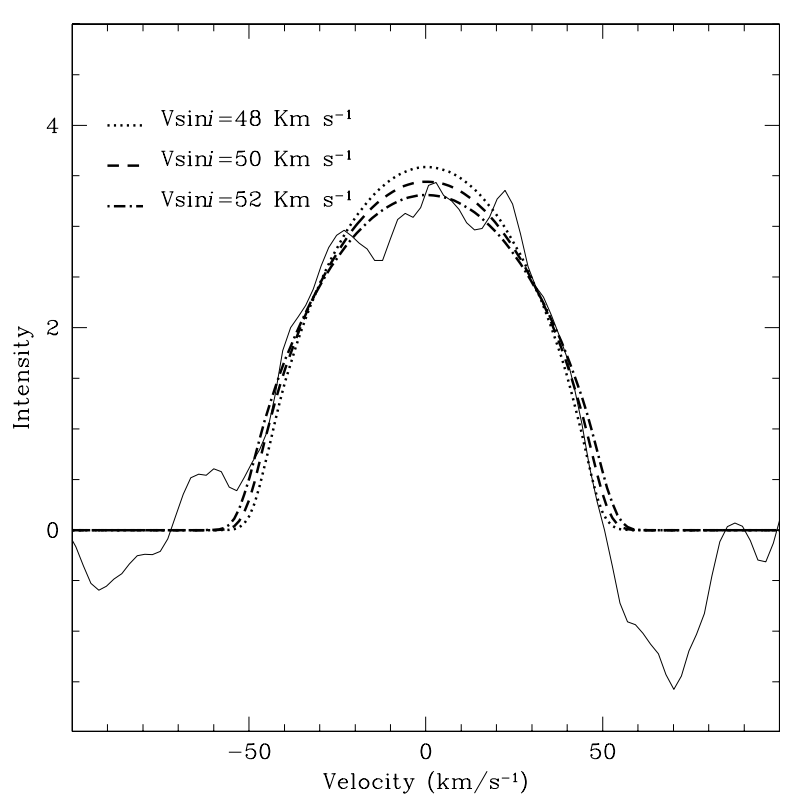

Fig. 2. Peak of the cross correlation between COM J1740-5340 and star \# 793 of G01 (solid line) in the spectral region included between $\lambda \lambda 4120-4180 \AA$, and examples of three possible choices of $V \sin i$ (broken lines): the best fit in this case turns out to be $51 \mathrm{~km} \mathrm{~s}^{-1}$.

velocities. The $V \sin i$, where $i$ is the orbit inclination, in the three spectral regions are (see Fig. 2) $51.0 \pm 1.5,49.0 \pm 1.5$, and $48.0 \pm 2.1 \mathrm{~km} \mathrm{~s}^{-1}$ respectively ( $1 \sigma$ errors); the weighted average is $V \sin i=49.6 \pm 0.9 \mathrm{~km} \mathrm{~s}^{-1}$. A fully compatible value ( $V \sin i=48.6 \pm 0.9 \mathrm{~km} \mathrm{~s}^{-1}$ ) has been obtained by subtracting quadratically the broadening terms due to the other factors mentioned above.

\subsection{Atmospheric parameters: Temperature and gravity}

The COM J1740-5340 effective temperature ( $\left.T_{\text {eff }}\right)$ has been determined using two different approaches: examining the $\mathrm{H} \alpha$ lines in our spectra (a reddening free method, see e.g., G01) or adopting the broad band colours resulting from the photometric observations of Kaluzny et al. (2003). Unfortunately, too few lines were available to derive the $T_{\text {eff }}$ from line excitation.

- The shape of the $\mathrm{H} \alpha$ absorption line wings is a good temperature indicator for $T_{\text {eff }}$ higher than about $5000 \mathrm{~K}$ (Fuhrmann et al. 1993); the core is best left out in all stars, and even more in our case, where an emission component is present (Paper II). Although this method assumes a "normal" atmosphere for COM J1740-5340, which is not strictly true (e.g., the star is not spherical), it is likely a good approximation for the deep regions where the $\mathrm{H} \alpha$ wings forms. Even if there are other Balmer lines in our spectrum, we worked only on $\mathrm{H} \alpha$ because it is less contaminated by metal lines, and less dependent on metallicity, gravity, and details in the convection treatment.

As a first evaluation, we tried to reproduce the $\mathrm{H} \alpha$ wings with synthetic spectra based on the appropriate Kurucz (1995) model atmosphere, without overshooting: we assumed $[\mathrm{Fe} / \mathrm{H}]=-2(\mathrm{G} 01)$, and $\log g=3.5$. Several different $T_{\text {eff }}$ 's

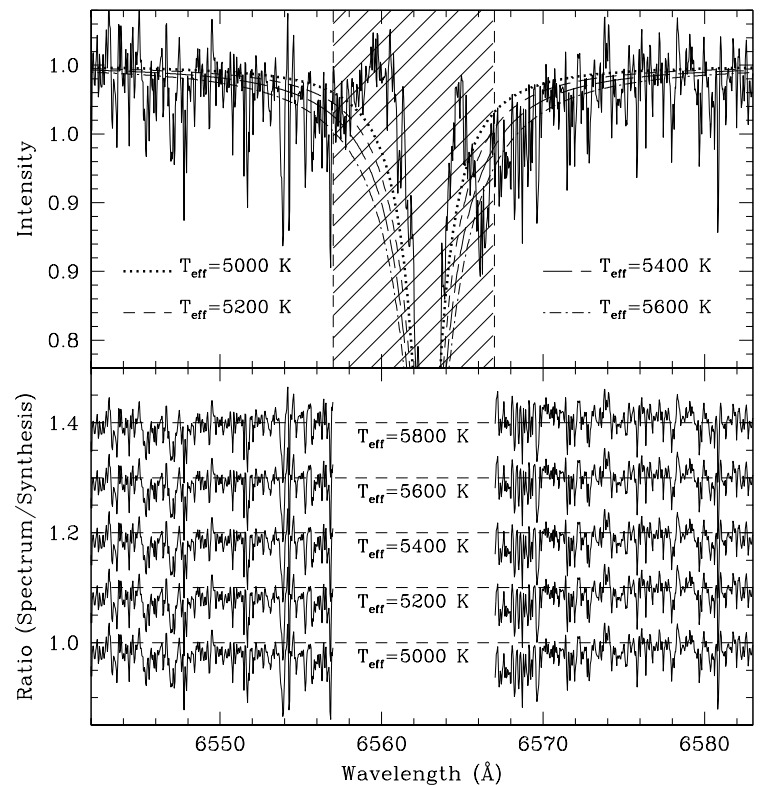

Fig. 3. Determination of effective temperature from the $\mathrm{H} \alpha$ wings. The upper panel shows the observed $\mathrm{H} \alpha$ spectrum and a set of synthetic Kurucz models at different $T_{\text {eff }}$ 's; the shaded region is excluded from the fit. The lower panel shows the corresponding ratios: the dashed horizontal lines represent ratio $=1$ for each case; note that the best fit cases are for $T_{\text {eff }}=5400$ and $5600 \mathrm{~K}$.

have been adopted (the cases at 5000, 5200, 5400, and $5600 \mathrm{~K}$ are shown in Fig. 3, top panel), and the best fit temperature appears to be $T_{\text {eff }}=5500 \pm 100 \mathrm{~K}$. The best fit temperature has also been determined by the ratio of the observed and synthetic profiles (Fig. 3, lower panel, where identity between the two profiles is indicated by the horizontal lines) yielding the same $T_{\text {eff }}$. In these estimates, the adoption of a metallicity is the most important factor, while the surface gravity impact on $T_{\text {eff }}$ is small, (an error of 0.3 dex on $\log g$ involves an error of $54 \mathrm{~K}$ on the evaluation of $T_{\text {eff }}$ ).

- The second method uses the photometric colours and implies independent knowledge of the reddening. Since the star is variable, we chose the average of the values published in the Table 1 of Kaluzny et al. (2003), obtaining $(B-V)_{\text {avg }} \sim 0.74$. We also assumed $E(B-V)=0.183$, as recently derived from Strömgren and Johnson photometry (Gratton et al. 2003). From the appropriate Kurucz transformation, we derived $T_{\text {eff }}=$ $5560 \pm 100 \mathrm{~K}$. We did not use $V-I$ for an independent determination, because we had only the Johnson-Cousins colour available, while Kurucz tables require both $V$ and $I$ in the Johnson system and this transformation is extremely uncertain in a $\mathrm{H} \alpha$ emitting star, like COM J1740-5340.

In summary, averaging the two temperatures, we obtain $T_{\text {eff }}=5530 \pm 70 \mathrm{~K}$, in good agreement with previous estimations (Ferraro et al. 2001; Paper I; Orosz \& van Kerkwijk 2003).

The surface gravity $g$ has been estimated from the position of COM J1740-5340 in the CMD and from its mass, as derived in Ferraro et al. (2001, 2003 respectively). Since the latter is constrained to be in the interval $0.22-0.32 M_{\odot}$, we took a value of $0.3 M_{\odot}$ and derived $\log g=3.5 \pm 0.2$, which is confirmed 
both by the following abundance analysis and the ionization equilibrium.

\subsection{Equivalent widths}

The equivalent widths $(E W \mathrm{~s})$ measured in our spectra have been used both for refining the set of atmospheric parameters (surface gravity from the equilibrium of ionization, and microturbulent velocity from elimination of trends with expected line strength) and for deriving the actual element abundances. For some elements (e.g., Li) and molecules $(\mathrm{CH})$ we also used spectral synthesis. A general problem encountered in the analysis is that the rotation washed out the weak, high excitation lines, the ones most directly depending on the element abundance. We are thus left with strong and saturated lines, whose analysis is dependent on the microturbulent velocity $v_{\mathrm{t}}$ and on the damping, respectively. For the latter we used the Barklem et al. (2000) treatment, the best available at the present time.

As a first step, $E W \mathrm{~s}$ were measured on the unidimensional, extracted spectral orders using a Gaussian function and an automatic routine working within the ISA package (Gratton 1988). A few lines, missed by the automated search, were added manually. Finally, the $E W \mathrm{~s}$ actually used for the abundance analysis were derived from the relation between line depth and equivalent width (for a detailed description of the procedure see Bragaglia et al. 2001). The list of lines measured and used in the abundance analysis is given in Table 1, together with the adopted excitation potentials (E.P. in $\mathrm{eV})$, the oscillator strengths ( $\log g f^{\prime}$ 's), the observed $E W$ in $\mathrm{m} \AA$ and the derived abundances. The typical error on the $E W \mathrm{~s}$ is about $5 \mathrm{~m} \AA$. Note that the measured $E W \mathrm{~s}$ are influenced by rotation since the line broadening often produces blends. A discussion of the line broadening relevance for determination of the elemental abundance is given in next section.

We measured lines both of Fe I and Fe II; from the equilibrium of ionization we obtained a surface gravity value $(\log g=$ $3.46 \pm 0.2)$ in perfect agreement with the one derived from photometry.

Optimization of the microturbulent velocity was done by comparing the abundances given by the intermediate strength lines with those derived from the strong ones, with well developed damping wings. In fact this would lead to a $v_{\mathrm{t}}$ quite smaller than required to get a good ionization equilibrium, so we compromised on an average value, adopting $v_{\mathrm{t}}=$ $1( \pm 0.5) \mathrm{km} \mathrm{s}^{-1}$.

In summary, the abundance analysis has been performed adopting the following atmospheric parameters: $T_{\text {eff }}=5530 \mathrm{~K}$, $\log g=3.46, v_{\mathrm{t}}=1.0 \mathrm{~km} \mathrm{~s}^{-1}$; by the analysis of $12 \mathrm{Fe}$ lines, we derive $[\mathrm{Fe} / \mathrm{H}]=-1.85 \pm 0.13$ (see next section for a better assessment of the errors). These values are to be compared with those used by G01 in the analysis of the NGC 6397 subgiants: $T_{\text {eff }}=5478 \mathrm{~K}, \log g=3.42, v_{\mathrm{t}}=1.32 \mathrm{~km} \mathrm{~s}^{-1}$ and $[\mathrm{Fe} / \mathrm{H}]=$ $-2.05 \pm 0.03$.

\section{The chemical abundance}

How do the element abundances in COM J1740-5340 compare with those measured in other stars of NGC 6397? To address
Table 1. Equivalent widths (only for the most reliable lines) measured on the COM J1740-5340 spectrum and derived abundances. Li I, Ti II, and Sr II abundances come from synthetic spectra (SS flag in the last column), while $\mathrm{Na}$ I abundances have been corrected for departure from local thermodinamical equilibrium (NLTE).

\begin{tabular}{|c|c|c|c|c|c|c|}
\hline Elem. & $\begin{array}{c}\lambda \\
(\AA)\end{array}$ & $\begin{array}{l}\text { E.P. } \\
(\mathrm{eV})\end{array}$ & $\log g f$ & $\begin{array}{r}E W \\
(\mathrm{~m} \AA)\end{array}$ & abund. & Notes \\
\hline Li I & 6707.80 & 0.00 & 0.19 & & 2.2 & SS \\
\hline $\mathrm{Na} \mathrm{I}$ & 5889.97 & 0.00 & 0.18 & 167.2 & 4.31 & NLTE \\
\hline $\mathrm{Na} \mathrm{I}$ & 5895.94 & 0.00 & -0.12 & 167.2 & 4.61 & NLTE \\
\hline Mg I & 5172.70 & 2.72 & -0.32 & 247.1 & 5.66 & \\
\hline Mg I & 5183.62 & 2.72 & -0.10 & 278.3 & 5.56 & \\
\hline Mg I & 5528.42 & 4.34 & -0.52 & 64.0 & 5.64 & \\
\hline $\mathrm{Ca} \mathrm{I}$ & 6122.23 & 1.89 & -0.27 & 82.0 & 5.07 & \\
\hline $\mathrm{Ca} \mathrm{I}$ & 6439.08 & 2.52 & 0.39 & 71.5 & 4.81 & \\
\hline Ti II & 4395.03 & 1.08 & -0.51 & & 3.4 & SS \\
\hline $\mathrm{Fe} I$ & 4045.81 & 1.49 & 0.28 & 317.2 & 5.79 & \\
\hline $\mathrm{Fe} I$ & 4063.59 & 1.56 & 0.06 & 267.1 & 5.91 & \\
\hline $\mathrm{Fe} I$ & 4071.74 & 1.61 & -0.02 & 227.9 & 5.87 & \\
\hline $\mathrm{Fe} I$ & 4202.04 & 1.49 & -0.71 & 124.7 & 5.74 & \\
\hline $\mathrm{Fe} I$ & 4383.56 & 1.49 & 0.20 & 275.7 & 5.68 & \\
\hline $\mathrm{Fe} I$ & 4404.76 & 1.56 & -0.14 & 192.9 & 5.73 & \\
\hline $\mathrm{Fe} I$ & 5325.19 & 3.21 & -0.10 & 85.0 & 5.84 & \\
\hline $\mathrm{Fe} I$ & 5405.78 & 0.99 & -1.84 & 82.5 & 5.48 & \\
\hline $\mathrm{Fe} I$ & 5434.53 & 1.01 & -2.12 & 62.5 & 5.29 & \\
\hline $\mathrm{Fe}$ II & 4923.93 & 2.89 & -1.35 & 91.8 & 5.89 & \\
\hline Fe II & 5018.45 & 2.89 & -1.22 & 95.4 & 5.84 & \\
\hline Fe II & 5316.62 & 3.15 & -2.02 & 52.7 & 5.81 & \\
\hline Sr II & 4077.71 & 0.00 & 0.17 & & 1.1 & SS \\
\hline Ba II & 6141.75 & 0.70 & 0.00 & 60.8 & 0.62 & \\
\hline
\end{tabular}

this question, we have considered three NGC 6397 SGB stars observed by G01 with a similar UVES configuration, and which display very similar atmospheric parameters (see Sect. 3.1). Combining the spectra of the three stars and convolving the summed spectrum with a rotational profile of $49.6 \mathrm{~km} \mathrm{~s}^{-1}$ (see Sect. 3.1), we have built a high $S / N$ template spectrum.

We can first exploit this template spectrum for assessing the typical uncertainty in our abundance analysis due to the effects of the rotation of COM J1740-5340. In fact, comparing the line $E W$ s both in the original SGB summed spectrum and in the spectrum broadened by rotation, we have noticed a systematic increasing in the abundances measured from the latter (even of $0.5 \mathrm{dex}$ ). This confirms that no immediate comparison should be done with the chemical composition of nonrotating stars, since COM J1740-5340 lines are broadened: blends with nearby lines can increase the $E W \mathrm{~s}$, and the derived abundances may be in error. Another very important factor is that the atmospheric model for COM J1740-5340 is not adequate, given the non spherical - and possibly variable geometry of COM J1740-5340: this mostly affects the ionized species, whose abundances are less reliable. From these consideration an error of 0.2 dex on the derived metallicity of $[\mathrm{Fe} / \mathrm{H}]=-1.85$ was considered, while the other abundances are determined with larger errors, about 0.3 dex. 

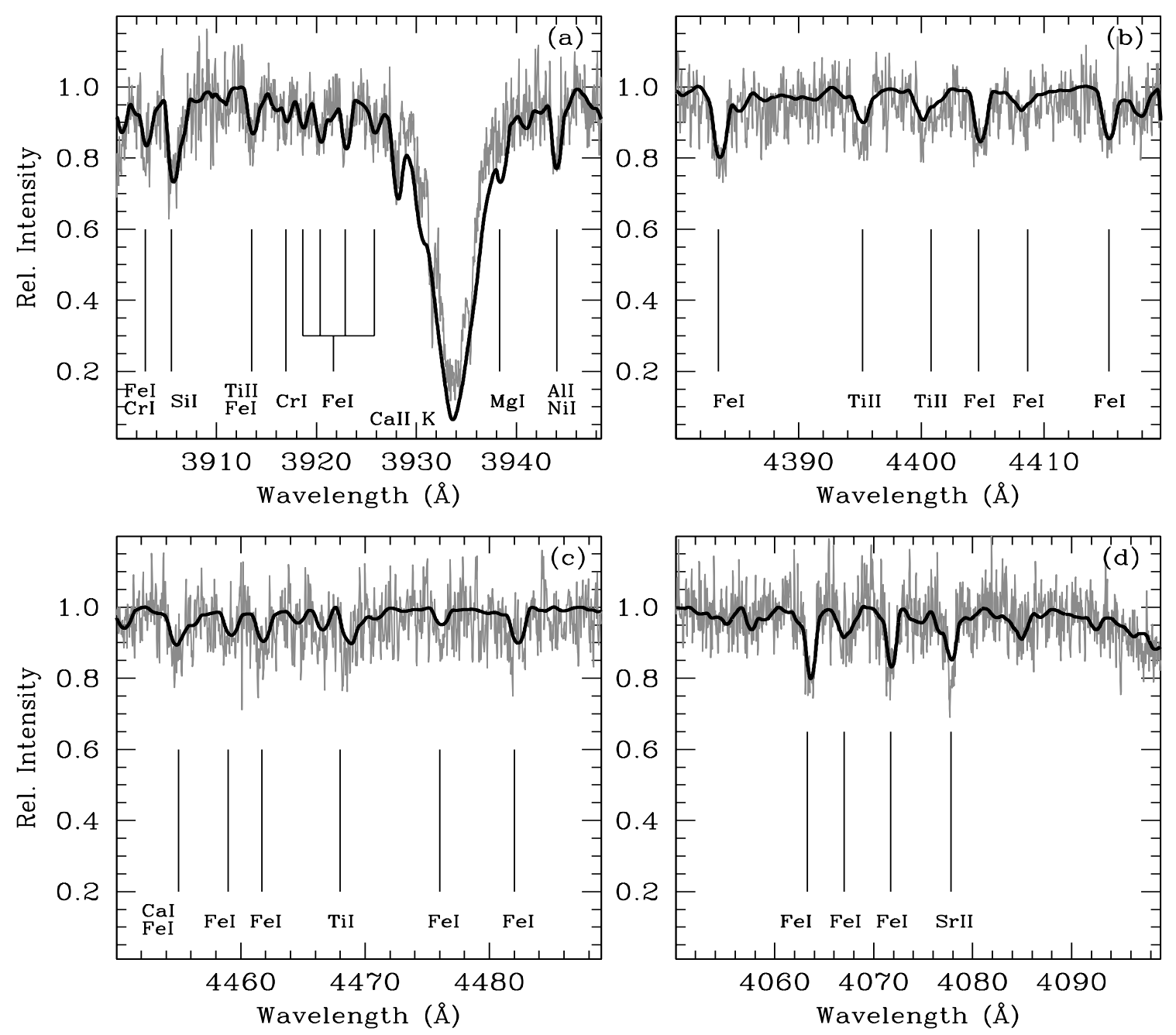

Fig. 4. Comparison between the COM J1740-5340 spectrum (grey line) and the subgiants template (black line, obtained by averaging, and broadening to account for rotation, three normal NGC 6397 subgiant stars, G01). We show the spectral regions near lines of Ca II (panel a)), Ti II (panel b)) Ti I (panel c)) and Sr II (panel d)). In all these regions many Fe I lines are also present.

Inspection of Fig. 4 (where they have been superimposed in four different regions comprising lines of $\mathrm{Ca}$, Ti, $\mathrm{Mg}, \mathrm{Sr}$ and $\mathrm{Fe}$ ) demonstrates that the COM J1740-5340 and the SGB template spectra show an excellent agreement, even if the COM J1740-5340 spectrum presents some evidences of lines filling (they are evident mostly in the core of the Ca II K line). Since the latter feature can be ascribed to the emission by the warmer region of the atmosphere (also responsible for the He I lines: see Papers I and II), this comparison supports the hypothesis that the chemical composition of COM J1740-5340 is similar to that of the SGB stars.

This conclusion would nominally contrast with the values collected in Table 2, where the forth column lists the differences between the average values of the abundances for each species measured in COM J1740-5340 and those derived by G01 for normal subgiant not rapidly rotating stars in NGC 6397. Taking these estimates at face value, COM J1740-5340 would show an overabundance of various species, particularly iron. However, according to the aforementioned difficulties involved in the analysis, we conclude that, within the errors, the abundances of $\mathrm{Fe}, \mathrm{Na}, \mathrm{Mg}$, and $\mathrm{Ti}$
Table 2. Abundances of COM J1740-5340 (in Col. 2), compared to those of normal SGBs; see G01 for Fe, $\mathrm{Na}$, and $\mathrm{Mg}$, while the other values are still unpublished (see text).

\begin{tabular}{lccc}
\hline \hline Element & $\begin{array}{c}\text { abund. } \\
\text { COM }\end{array}$ & $\begin{array}{c}\text { abund. } \\
\text { SGB }\end{array}$ & $\Delta$ (abund.) \\
\hline $\mathrm{Fe}$ I & 5.70 & 5.42 & +0.28 \\
$\mathrm{Fe}$ II & 5.84 & 5.26 & +0.58 \\
$\mathrm{Li}$ I & 2.2 & 1.2 & +1.00 \\
$\mathrm{Na}$ I & 4.46 & 4.51 & -0.05 \\
$\mathrm{Mg}$ I & 5.62 & 5.70 & -0.08 \\
$\mathrm{Ca}$ I & 4.94 & 4.54 & +0.40 \\
$\mathrm{Ti}$ II & 3.4 & 3.39 & 0 \\
$\mathrm{Sr}$ II & 1.1 & 0.75 & +0.35 \\
$\mathrm{Ba}$ II & 0.62 & -0.06 & +0.68 \\
\hline
\end{tabular}

in COM J1740-5340 are compatible with those measured in normal NGC 6397 stars. 

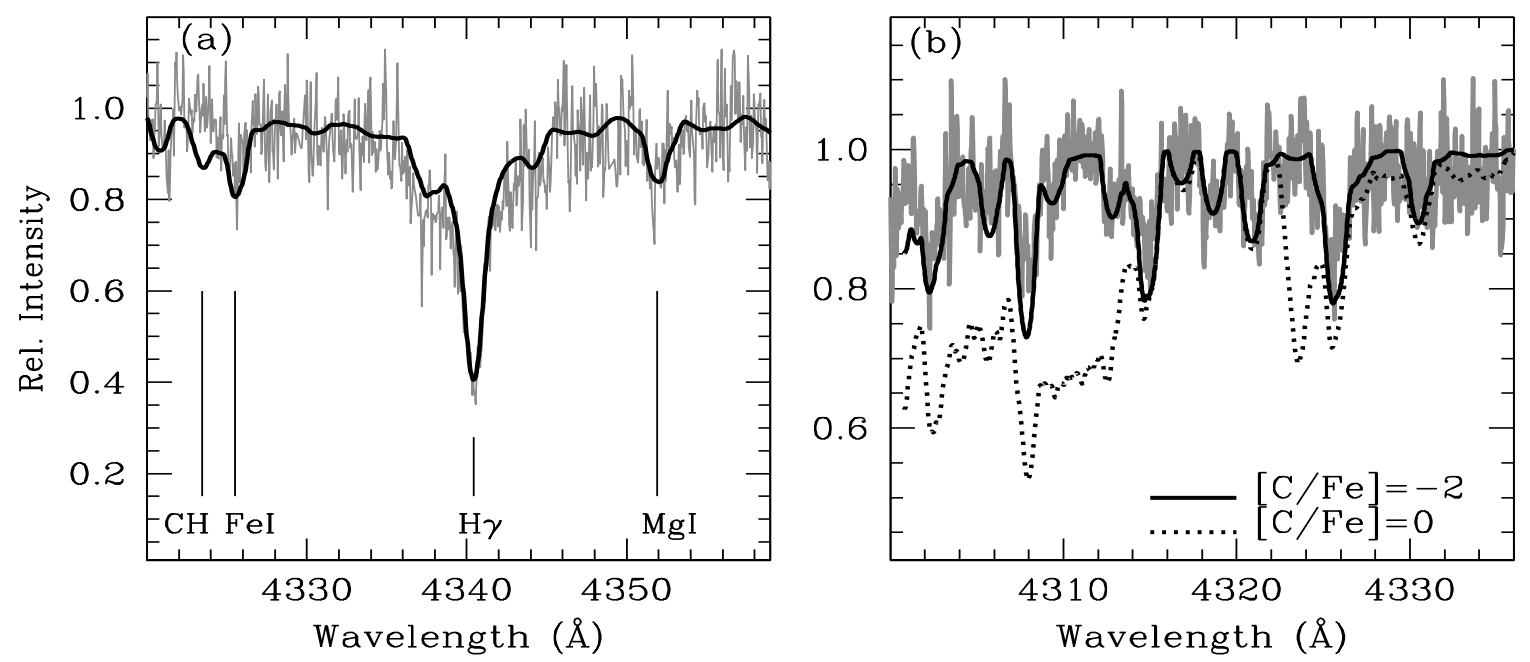

Fig. 5. Evaluation of the carbon abundance from the $\mathrm{CH}$ band. Panel a) shows a clear depletion in the region of the $\mathrm{CH}$ band in the COM J1740-5340 spectrum (grey line) with respect to the NGC 6397 subgiants template (black line). Panel b) shows the result of spectral synthesis, demonstrating that $\mathrm{C}$ is strongly underabundant in the COM J1740-5340 atmosphere.
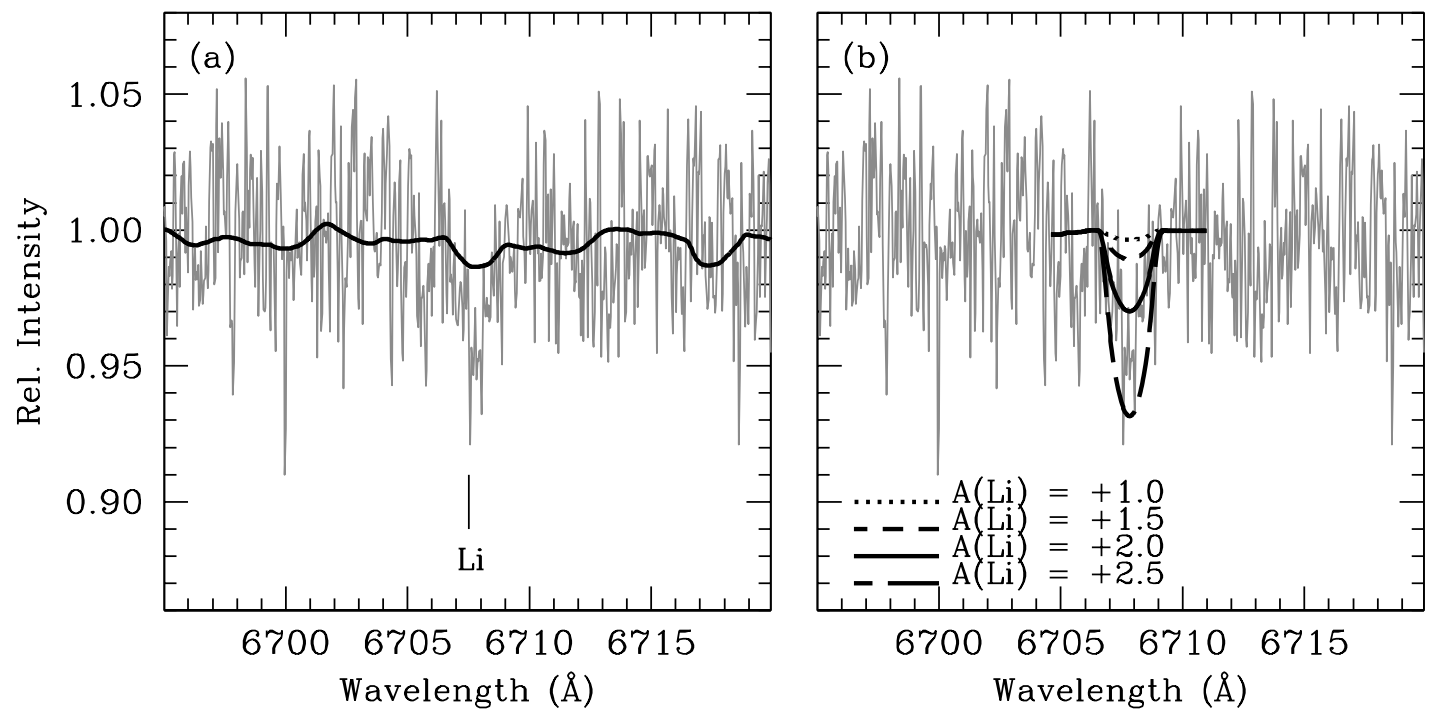

Fig. 6. In panel a) we compare the COM J1740-5340 spectrum with the NGC 6397 subgiants template: the Li I line is clearly deeper than in the template. In panel b) we show a comparison with four different synthetic spectra, demonstrating the high Li content.

\subsection{Discrepant elements: $\mathrm{Ca}, \mathrm{C}, \mathrm{Li}$}

In the previous section we have shown that, once the broadening effects on $E W$ s are taken into account, the chemical abundances of COM J1740-5340 are fairly similar to those of SGB stars. Nevertheless, even after adjustment for rotation, some elements still show disagreement in abundances with the other NGC 6397 stars. Since for the Sr II and Ba II only one line (coming from ionized species, whose abundance determination is less reliable) each is present in our spectra, we do not consider here these two elements, and focus only on the three others.

(i) $\mathrm{Ca}$ : We measured only four $\mathrm{Ca}$ I lines in our spectra, among which are the very strong ones at $\lambda \lambda 4226.74 \AA$ and $6162 \AA$. Since the strength of these lines depends more on $v_{\mathrm{t}}$ than on abundance (hence they are rarely used), we retained only the two most reliable for abundance determination (namely at $\lambda \lambda 6122 \AA$ and $6439 \AA$ ). We accurately checked our spectrum near the lines for cosmic rays and hidden blends, and directly compared it to the SGB template. COM J1740-5340 $\mathrm{Ca}$ I lines appear deeper than in the template, as a conseguence there are differences in the $E W$ s (e.g., about $20 \mathrm{~m} \AA$ for the $6122 \AA$ line). Taking into account the slightly different atmospheric parameters for COM J1740-5340 and the SGB template, this implies a $\mathrm{Ca}$ I abundance about 0.4 dex higher for our star.

(ii) C: Comparing our spectrum to the template SGB one, it is clear that $\mathrm{C}$ is strongly underabundant in COM J1740-5340 (Fig. 5a). To get a quantitative estimate of the $\mathrm{C}$ abundance we have built synthetic spectra for the region near the $\mathrm{CH} G$-band (around $4310 \AA$ ) by assuming $[\mathrm{C} / \mathrm{Fe}]=-2,-1,0,+1$ : two of them are shown in Fig. 5b. The result is that COM J1740-5340 spectrum is fully compatible with complete absence of $\mathrm{C}$ in the atmosphere. This would indicate a composition resulting from 
the $\mathrm{CN}$ cycle at equilibrium, when most $\mathrm{C}$ has been burned to $\mathrm{N}$; this evidence suggests that COM J1740-5340 could be a deeply peeled stars.

Ergma \& Sarna (2003) computed the evolution of the surface abundances for a few key elements in two cases proposed for explaining the nature of COM J1740-5340 (low mass bloated main sequence star or evolved star). From their calculations $\mathrm{C}, \mathrm{N}$, and $\mathrm{O}$ would behave like in "normal" metal poor stars if COM J1740-5340 is a low mass main sequence star. On the contrary, only $\mathrm{O}$ would have a normal abundance, while $\mathrm{N}$ would be overabundant, and $\mathrm{C}$ underabundant in the case of a heavier, evolved star which has lost mass. Unfortunately, no $\mathrm{O}$ and $\mathrm{N}$ lines are present in our spectra, but the very low $\mathrm{C}$ abundance favours the second possibility.

(iii) $\mathrm{Li}$ : The $\mathrm{Li}$ I resonant doublet at $6708 \AA$ is much stronger in our spectrum than in the normal NGC 6397 subgiants: Fig. 6a shows a comparison between COM J1740-5340 and the SGB template, while Fig. $6 \mathrm{~b}$ is a zoom in on the Li feature with a few synthetic models superimposed. From the best fit synthetic spectrum we obtained a $\mathrm{Li}$ abundance $(A(\mathrm{Li})=$ $2.2( \pm 0.2)$ in the usual notation), which is quite surprising. In fact, the $\mathrm{Li}$ abundance of turn-off stars, i.e. of unevolved stars with the original abundance, is $A(\mathrm{Li})=2.34 \pm 0.08$ (Bonifacio et al. 2002), while subgiant stars, which have already diluted their original $\mathrm{Li}$, have $A(\mathrm{Li}) \sim 1.5$ (Castilho et al. 2000), a value also confirmed by $A(\mathrm{Li}) \sim 1.2$ measured in the three SGB stars used as template (see Table 2).

This value is at odds with what is expected both from a $\sim 0.3 M_{\odot}$ main sequence star recently acquired in the binary system (since low mass stars are completely convective and the original surface $\mathrm{Li}$ has long been transported to the interior and there destroyed) and from a heavier star peeled by mass loss down to the zone where the $\mathrm{CN}$ cycle is active (incomplete $\mathrm{CNO}$ process, burning $\mathrm{C}$ to $\mathrm{N}$ ). How can we explain this high Li content? It cannot be the original, cosmological Li, nor Li produced by mechanisms such as the Hot Bottom Burning, which works in intermediate mass stars (Ventura et al. 2001). Planet engulfing (Alexander 1967; Siess \& Livio 1999) is also improbable: the orbit of a planet around COM J1740-5340 could not be stable and $\mathrm{Li}$ would not be the only element affected (e.g., C too would be enriched, not depleted).

At the moment the most plausible explanation could be fresh $\mathrm{Li}$ production due to nuclear reactions occurring on the stellar surface, since a nearby source of cosmic rays (the pulsar) is available. In particular, it should be noticed that a peeled star, where we see at the surface the products of incomplete $\mathrm{CNO}$ cycle, should have a large atmospheric abundance of ${ }^{3} \mathrm{He}$ : ${ }^{7} \mathrm{Li}$ can be easily produced by a cosmic ray flux containing $\alpha$-particles (D'Antona, private communication). Moreover, at the very high temperature required (a few million $\mathrm{K}$ ), the star would also be an X-ray source, and this is actually observed (Grindlay et al. 2001, 2002), even if the X-ray emission can be also attributed to the interaction between the relativistic wind from the MSP and the mass lost from the companion. Finally, we recall that $\mathrm{Li}$ enhancement, found in some chromospherically active binary systems, is actually attributed to nucleosynthesis in the corona, i.e., to a similar mechanism (see, e.g. Montes et al. 1997, 2000 for a review).

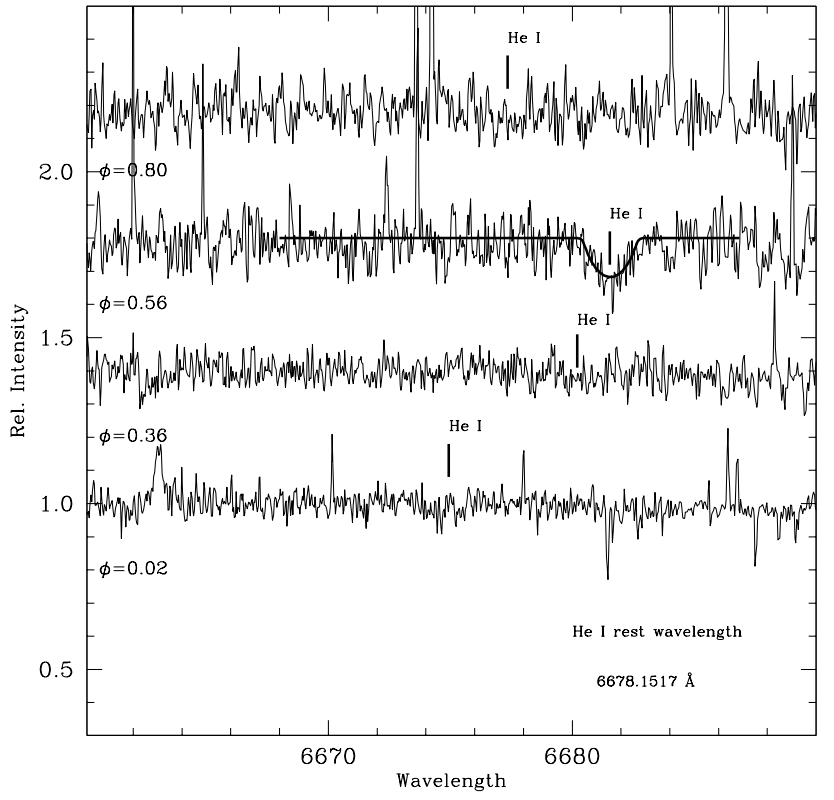

Fig. 7. He I line at $\lambda 6678 \AA$ at different orbital phases. The heavy solid line overlaid on the spectrum acquired at phase $\phi=0.56$ (quadrature) is an empirical profile broadened by stellar rotation. In each spectrum the He I position is indicated.

\subsection{He I at $\lambda 6678 \AA$}

As discussed in Papers I and II the inspection of the spectral data-set reveals the unexpected presence of $\mathrm{He} \mathrm{I}$ lines at $\lambda 5876 \AA$ and $\lambda 6678 \AA$. In particular the He I properties at $5876 \AA$ were fully described in Paper II. Here we present the phase variations of the $\mathrm{He}$ I line at $6678 \AA$ (see Fig. 7). As can be seen from the figure, such line is clearly visible (and measurable) only at $\phi=0.56$ (quadrature). Note that at such a phase the line has the same $E W$ as the line detected at $5875 \AA$ ( $0.19 \AA$ see Paper II, Fig. 4). At the other orbital phases the $S / N$ is not good enough to allow a reliable evaluation of the line $E W$. At $\phi=0.36$ (near conjunction) the line is practically absent. This in not a surprising result since at this phase also the $5878 \AA$ line is shallower than at the other phases. At $\phi=0.02$ (quadrature) the He line is very shallow; moreover a cosmic ray was present just at the He I wavelength and, even if we removed it, it is possible that the line is still partially contaminated by this cosmic ray. Spectra at higher $S / N$ are required to clearly assess the presence of the line at this phase.

\section{Summary and conclusions}

We have presented the chemical composition of COM J1740-5340, the non-degenerate companion to the millisecond pulsar PSR J1740-5340 in NGC 6397. Abundance analysis of high resolution spectra has allowed us to determine the abundance of iron $([\mathrm{Fe} / \mathrm{H}]=-1.85 \pm 0.2)$ and several other elements. The general conclusion is that the abundance is fully compatible with that of normal, single stars in NGC 6397, with a few notable exceptions (Ca, C, and $\mathrm{Li}$ ) that may be attributable to the peculiar history of the star, subject to (extreme) mass loss and interactions with the millisecond pulsar. In particular, the strong $\mathrm{C}$ depletion seems 
to indicate that COM J1740-5340 is not a perturbed low mass main sequence star, but had instead a larger mass and has been peeled down to the present $\sim 0.3 M_{\odot}$. Future observations will allow the appropriate measure of $\mathrm{N}$ and $\mathrm{O}$ abundances, and would give further support to this hypothesis, if those elements are found overabundant and unchanged, respectively, with respect to other stars in NGC 6397.

From the derived chemical composition, we do not see any indication of accretion of elements from the type II supernova explosion that left behind the neutron star (e.g., $\mathrm{Mg}$ ), since the composition of COM J1740-5340 is mostly similar to the other stars in the cluster. Several scenarios, among which we are not able to discriminate with the present data, are possible: i) the secondary has been acquired by the system only after the SN (star exchange in a collision); ii) the $\mathrm{SN}$ wind was too fast for the secondary star to accrete a significant amount of ejected material; iii) and/or the neutron star has had enough time to remove all the accreted material, together with a fair fraction of its own original mass, from the companion.

Acknowledgements. We thank E. Carretta and L. Cinque for useful comments and discussions. This research was supported by the Agenzia Spaziale Italiana (ASI).

\section{References}

Alexander, J. B. 1967, The Observatory, 87, 238

Ballester, P., Modigliani, A., Boitquin, O., et al. 2000, The Messenger, 101,31

Barklem, P. S., Piskunov, N., \& O’Mara, B. J. 2000, A\&AS, 142, 467

Bonifacio, P., Pasquini, L., Spite, F., et al. 2002, A\&A, 390, 91

Bragaglia, A., Carretta, E., Gratton,, R. G., et al. 2001, AJ, 121, 327

Castilho, B. V., Pasquini, L., Allen, D. M., Barbuy, B., \& Molaro, P. 2000, A\&A, 361, 92

D’Amico, N., Lyne, A. G., Manchester, R. N., Possenti, A., \& Camilo, F. 2001a, ApJ, 548, L171

D'Amico, N., Possenti, A., Manchester, R. N., et al. 2001b, ApJ, 561, L89

D'Antona, F. 1996, in Evolutionary Processes in Binary Stars, ed. R. A. M. J. Wijers, \& M. B. Davies (Dordrecht: Kluwer), 477, 287
Edmonds, P. D., Gilliland, R. L., Camilo, F., Heinke, C. O., \& Grindlay, J. E. 2002, ApJ, 579, 741

Ergma, E., \& Sarna, M. J. 2003, A\&A, 399, 237

Ferraro, F. R, Possenti, A., D’Amico, N., \& Sabbi, E. 2001, ApJ, 561, L93

Ferraro, F. R., Sabbi, E., Gratton, R. G., et al. 2003, ApJ, 584, L13 (Paper I)

Fuhrmann, K., Axer, M., \& Gehren, T. 1993, A\&A, 271, 451

Gratton, R. G. 1988, Rome Obs. Preprint Ser. 29

Gratton, R. G., Bonifacio, P., Bragaglia, A., et al. 2001, A\&A, 369, 87 (G01)

Gratton, R. G., Bragaglia, A., Carretta, E., et al. 2003, A\&A, 408, 529

Gray, D. F. 1992, The Observation and Analysis of stellar Photosphere (Cambridge Univ. Press)

Grindlay, J. E., Heinke, C. O., Edmonds, P. D., Murray, S. S., \& Cool, A. M. 2001, ApJ, 563, L53

Grindlay, E., Camilo, F., Heinke, C.O., et al. 2002, ApJ, 581, 470

Grundahl, F., Vandenberg, D. A., Stetson, P. B., Anderson, M. I., \& Briley, M. 2000, in The Galactic Halo: from Globular Clusters to Field Stars, ed. A. Noels, P. Magain, D. Caro, et al., 503

Hansen, B. M. S., \& Phinney, E. S. 1998, MNRAS, 294, 569

Kaluzny, J, Rucinski, S. M., \& Thompson, I. B. 2003, AJ, 125, 1546

Kurucz, R. L. 1995, CD-ROM 13, Opacities for Stellar Atmospheres: Abundance Sample (Cambridge: Smithsonian Astrophys. Obs.)

Lucatello, S., \& Gratton, R. G. 2003, A\&A, 406, 691

Montes, D., Fernández-Figueroa, M. S., De Castro, E., et al. 2000, A\&AS, 146, 103

Montes, D., Fernández-Figueroa, M. S., De Castro, E., \& Sanz-Forcada, J. 1997, A\&AS, 125, 263

Orosz, J. A., \& van Kerkwijk, M. H. 2003, A\&A, 397, 237

Podsiadlowski, P. 1991, Nature, 350, 136

Podsiadlowski, P., Rappaport, S., \& Pfahl, E. D. 2002, ApJ, 565, 1107

Possenti, A. 2002, in WE-Heraeues Seminars on Neutrin Stars, Pulsars and Supernova remnants, MPE Report 278, ed. W. Becker, H. Lesch, \& J. Trumper, 183

Sabbi, E., Gratton, R. G., Ferraro, F. R., et al. 2003, ApJ, 589, L41 (Paper II)

Siess, L., \& Livio, M. 1999, MNRAS, 308, 1133

Stappers, B. W., van Kerwijk, M. H., Bell, J. F., \& Kulkarni, S. R. 2001, ApJ, 548, L183

Tauris, T. M., \& Savonije, G. J. 1999, A\&A, 350, 928

Taylor, J. M., Grindlay, J. E., Edmonds, P. D., \& Cool, A. F. 2001, ApJ, 553, L169

Ventura, P., D’ Antona, F., Mazzitelli, I., \& Gratton, R. 2001, ApJ, 550, 65 